\title{
Research on Key Technologies and Application of Vehicle Networking Zunyi Shang ${ }^{1, a}$, Hang Su, b and Pengfei Yin ${ }^{1, c}$ \\ ${ }^{1}$ Network and Information Center, Dalian Jiaotong University, Dalian 116028, Liaoning, China \\ aszy@djtu.edu.cn, bsuh@djtu.edu.cn, cypf@djtu.edu.cn
}

Keywords: Vehicle networking; Intelligent transportation; Internet of things; Key technology Application

\begin{abstract}
In recent years, the birth of vehicle networking has brought great hope to intelligent transportation industry, but also g plays an increasingly large role gradually in people's daily life. This paper firstly introduces the components of the vehicle networking and its birth and development process, and researches on its wireless network integration technology, automatic recognition technology, information intelligent processing technology, in the end, looks ahead the application prospect of vehicle networking in the development of intelligent transportation and intelligent city.
\end{abstract}

\section{Introduction}

With the rapid development of modern life, the demand for vehicles being more intense, popularity rate of Passenger cars to enter the usual family is more and more higher. According to statistics, the total amount of China's family car is a very huge, has exceeded the mark of 150 million. But more and more cars cause insurmountable bottleneck for the city to solve the problem of traffic congestion, and constantly plagued the government transportation department and traveler. Earlier in 2015, the concept of Internet of things has been put up in the world of information society, in recent years, with the development of technology of Internet of things and Promotion of application in some fields, vehicle networking came into being making the concept of information networking, intelligent into heart. In the meanwhile, People also want .car networking in intelligent transportation, smart city and other areas to inspire greater development potential.

Construction Elements of Vehicle Networking. Vehicle networking mainly consists of several levels, advanced sensor technology level, network coverage technology, wireless network communication technology, automatic recognition and intelligent processing technology, etc. a large amount of data of sensors, intelligent vehicle sensors gathering ,processing, transmission and sharing, making the car and the people, the car and the urban transportation system, the vehicle and the city network system's interconnected, by the network to realize the intelligent identification, tracking and positioning, monitoring and control of terminal information. According to the function of the vehicle network system, the network can be divided into three components, the sensing layer, the application layer and the network layer 


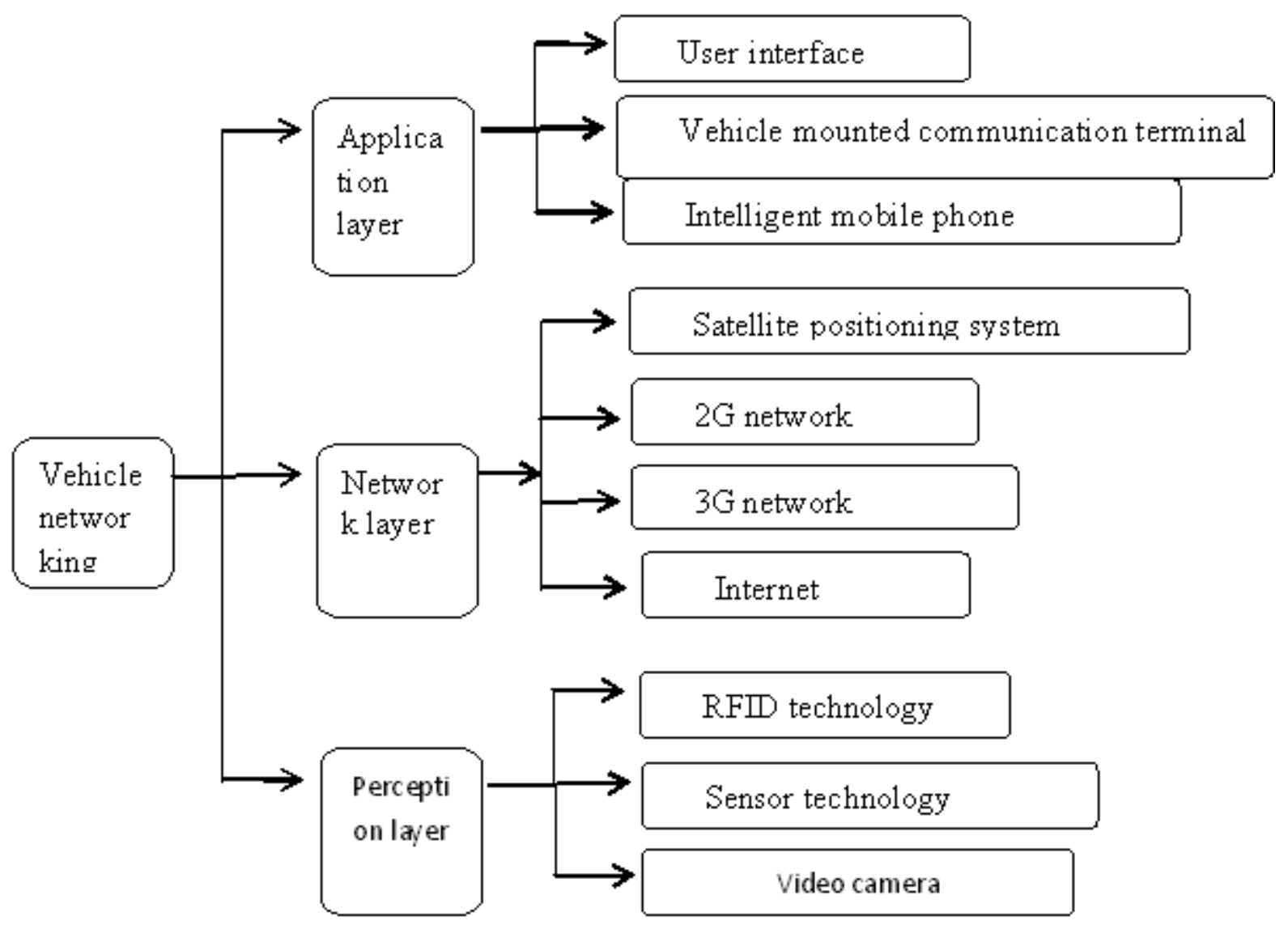

Figure 1. Construction elements of the car networking

Application Layer. Man-machine communication is the main application layer to play in the car networking in, like a wide variety of terminal equipment, on-board microcomputer system and the central control system, etc. The core elements of the network is the electronic information navigation, GPS satellite positioning, intelligent vehicle control system, voice identification and mobile wireless network technology constituting vehicle navigation information system of communication network. The system model can be through the network to the passenger car drivers and passengers to push the comprehensive urban transportation, life and other convenient information and services.

Network Layer. Network layer is the key layer in vehicle network, by the wireless communication network, intelligent control algorithm, GPS positioning system to achieve Internet connection, to achieve a various acquisition, processing, transmission of information and electronic data, so to make the ultra-long range of communications and control become possible.

Perception Layer. The role of the perception layer in the car network is to use to electronic tags (RFID) and readers, various sensor components (Such as temperature measuring instrument, speed measuring element, road monitoring probe, etc.) video surveillance video probe to do A large number of data on the mobile phone task and, and properly processed and uploaded the vehicle information, traffic system the weather conditions obtained, the complex data to the network.

\section{The Concept of Vehicle Networking}

The rapid development of the Internet of things is also an important factor in the emergence of the car networking. The definition everyone hold of car networking being different in the intelligent transportation technology, vehicle networking is a scientific and reasonable integration with advanced information and communication technology, sensor collection technology, intelligent 
technology and information digital processing technology, applying to the overall road traffic management system to Consist of a large area, covering the comprehensive real-time region, accurate and fast the whole system; Definition of network and communication at the end of the vehicle, Car networking is noun developing and combined of the industrial automation industry and wireless network communication industry rapidly, which Is mainly not fixed form with vehicular wireless apparatus interface structure. The wireless interface terminal can not only connect to the homogeneous or heterogeneous network, but also can be connected to the fixed communication facilities on the ground to realize the communication between the vehicle and the vehicle, the vehicle and the ground.

The definition of each person is different, but there are also similarities between them: Firstly, advance information digital technology including sensor collection technology, Internet technology, computer algorithm control technology, intelligent automation technology, makes the vehicle, the person, the ground, the transportation facility and so on form a unified comprehensive system to satisfy the traffic safety, traffic management, urban services and Internet surfing and other functions in order to improve the travel efficiency, reduce the risk of traffic safety, ease urban traffic pressure purposes.

\section{The Key Technology of Vehicle Networking}

At present, with more and more countries studying the vehicle networking technology, The United States, Japan and other countries are investing heavily in new technology research and development. Our country's urban traffic pressure increasing year by year, our country also thinks high of the development of the car Networking Industry. In October 2010, at the international networking summit in Wuxi, the car networking has become the third major projects of China's major projects, having received support from the state and the government. I think, car networking core technology is the communication and the core technology of vehicle terminal communication equipment, RFID technology, GPS positioning technology, wireless network technology, intelligent technology, big data cloud computing technology, automatic identification technology, etc.

Introduction of RFID Technology. RFID is a kind of automatic identification technology of distance and uses radio frequency digital communication to get familiar with the object and get the data of the object. RFID is also a kind of simple wireless communication system including base units of an electronic tag, reader, to locate, track and control target. In recent years, RFID technology has been developed in many industries, which is convenient for people's life, like the management of tourist attractions and parking lots, the monitoring of power lines and pipelines, etc. RFID has many advantages such as identification of multiple mobile targets one time, radio frequency reading and writing of the distance, memory capacity is relatively large, small size, high reliability, convenient, high adaptability, etc. So RFID is a wide range of applications in all aspects of the car networking technology

The Technology of GPS Positioning and Navigation. The technology of GPS positioning and navigation which is provided by American to launch the satellite is positioning and navigation system with high accuracy, full working time, intelligent, real-time and so on. As long as the customer has networked terminal equipment, you can use the location tracking, and navigation function. So, in the building and realizing process of vehicle networking, The technology of GPS positioning and navigation is an integral part.

Wireless Network Technology. Wireless network technology as the name suggests, is working in the wireless signal environment. This feature makes it simple, reliable, fast, low cost. So, wireless network technology is very common in the car networking application, like all kinds of company's 4G, 3G technology, WLAN technology, UWB technology, etc., which makes car networking and the Internet achieve docking, Therefore, In the future development of the car networking, wireless technology and sensor acquisition information technology playing a part together other, when the vehicle traveling to the corner and other sensing devices, the wireless communication can be turned on. Besides, because the number of collected information by vehicles 
is huge, also need to use the communication technology to send a huge amount of information data to the internet, to Use the cloud computing technology of the Internet to carry on processing. So, wireless network technology is a key technology in vehicle networking.

Intelligence and Identification Technology. In the building of vehicle networking, the final goal is to realize the external connection of the vehicle and the function of automatic driving, so you should add some scientific and advanced intelligent technology in the development process (such as Artificial intelligence control, Cluster algorithm control, technology Intelligent BMS etc.) to Let the car have the function of automatic identification and judgment, more intelligent operation. What's more, the technology automatic identification is a necessary part in vehicle networking which mainly is used in automobile anti-theft and safety function to identify the car owner and the drivers and through the vehicle networking technology will send personnel status information and real-time feedback to the network, to facilitate the access of personnel to view, which is also an important means to regulate traffic operations management.

Computer Cloud Computing Technology. Computer cloud computing technology is a key technology to achieve through the Internet technology. Through the Internet, divide the information data into a number of small unit data, and then send to a huge computing system constituted of multiple servers on the Internet. By looking up each server, and analyzing and identifying, the results will be returned to the user, so that the technology of solving the problem is cloud computing technology. This mature key technology can be in a few seconds, through the Internet server to complete the operation of the unit or the millions of information data, and can have the same high computing efficiency. In networking technology, information collected by a large number of vehicles being very huge, but also quite complex, Cloud computing technology can be said to be the best solution to the problem, with Cloud computing technology, the car network vehicle information, traffic conditions, urban services such as all kinds of information and data can be one easy to solve.

The Difference of Car Networking and Other Network System. Other management system used in transportation management departments and vehicle networking is different (Such as intelligent traffic management system), whose own advantages can make the car networking to achieve a more systematic perception, a wide range of docking and ultra-efficient digital processing and use of information, so, The development of vehicle networking is the urgent demand and the best result of traffic management system and is the best way to improve the traffic capacity of the city.

Vehicle Networking Completely Manages Vehicles. Vehicle mounted communication terminal is the best equipment for vehicle networking to manage vehicles whose operation makes it possible to make the network real-time monitoring and automobile traffic forecast possible, not only to ensure the passage of each road, but also to avoid traffic congestion and accidents. In addition, the electronic tag of the mobile car which is the same as the person's identity card is unique, representing all the information of the vehicle. Vehicle driving route, stop location and other information help to ride in network construction and can effectively reduce the pressure on urban traffic, empty driving rate and the urban resources waste, but also reduce the cars on the road and the city key business district space occupancy. The most important is to reduce the emission of automobile exhaust, which is conducive to energy conservation and emission reduction, improving air quality, so that the city home to become more beautiful.

Car Networking Makes Travel Services More Colorful. The emergence of the car networking makes travel become more relaxed and convenient. In the past, people always do ell preparing for a vehicle travel before it goes out. Car networking making all simple, the vehicle information terminal equipment timely upload car information to network which let us stay at home and know the reality of the vehicle. After having vehicle networking, we can get on the bus and then do a new understanding of the road. Through voice communication and network push service, we can know the best route of traffic, and passengers can also through the on-board equipment connect to the Internet, enjoy the Internet service brought to us, let you work in the travel journey, entertainment two not mistake, let your driving on the way to become more rich and colorful. 
Car Networking Promote the Development of Other Related Industries. Car networking operations can be said to be the result of the results of multiple industries. There being IT industry, car manufacturers, communications companies, and computer intelligence, etc. The bright prospects of car networking make all walks of life see the benefits of their own development, in the process of serving to the vehicles networking, so that enterprises unconsciously improve the development of their own enterprises. Besides, in the development of the car networking, there are many technical difficulties and key issues needed to be broken, For example, sensor signal acquisition technology, wireless communication technology, satellite positioning and navigation technology, and other key technologies. Now satellite positioning technology having been basically mature, 3G, 4G network having covered the whole country, car networking and integration of various industries having been very successful, It is expected that the next three years, the value of the car networking industry can reach 100 billion, which is a good momentum of development, and can bring a lot of jobs.

\section{Car Networking Application Case in the Future}

End equipment (such as the Apple Corp) in the future, a key function is to develop vehicle information release software. First of all, it can make the phone through the APP application software into the car keys, through the Wi-Fi signal or the launch of the mobile phone network signal to open or close the car door lock, can also through the phone's interface, monitor the state of the vehicle, the operation of electric vehicles for battery charging or early open air conditioning equipment, etc. Secondly, in the course of vehicle driving, the traffic information punish by networking can be received through the wireless signal, Simultaneously, also can percept the situation inside and outside the vehicle in cooperation with the vehicle sensing equipment, using car screen to display early warning information, driving vehicle safely. In addition, we can develop voice communication software and terminal equipment, to achieve the vehicle and human intelligent dialogue, to facilitate the driving. Finally, the vehicle can install some entertainment terminal equipment, so that passengers can travel on the way to surf the Internet and can also use the WiFi series technology to share online games with riders or driving partners. In a word, once similar software developed, in the case of a good application prospects, believe that a variety of colorful car equipment and software will be endless.

\section{Summary}

The development of vehicle networking is the urgent demand and development direction of China's transportation industry, is also a typical representative in the development model of the new Internet of things in China. Although there are still many technical problems need to overcome and various core technologies to be improved in the development of the car networking, But we assert that in the near future, with the Internet of things technology, cloud computing technology, signal acquisition and processing technology, vehicle manufacturing technology, car networking will have a better application prospects, a more broad space for development.

\section{References}

[1] Zhao Jing, Yu Xiao hong, Huang Bo and so on, the structure system of the Internet of things and the development of Inernet [J]. Communication technology, 2010, 43 (09): 106-108.

[2] Zhu Tong yu, Wang Jia chuan, Chen Zhi hong, first research on vehicle networking technology [J].traffic engineering, 2011,77 (05): 266-268.

[3] Liu Fuqiang, Shan Lian hai the research on the wireless network architecture of vehicle mounted mobile heterogeneous and key technology of [J]. ZTE technology, 2010 (03): 47-51.

[4] Liu Xiaoyang, Wu Minyou. Car Networking: application of the Internet of things in city traffic network [J] Computer applications, 2012, 32 (4): 900-904. 